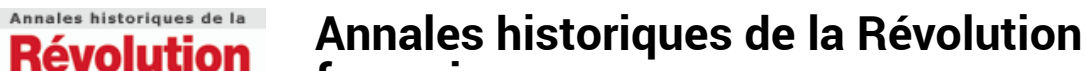 \\ française française
}

340 | avril-juin 2005

Les arts et la révolution

\section{Martin Guillaume Biennais : une carrière exceptionnelle}

Anne Dion-Tenenbaum

\section{(2) OpenEdition \\ 12 Journals}

\section{Édition électronique}

URL : https://journals.openedition.org/ahrf/2058

DOI : 10.4000/ahrf.2058

ISSN : 1952-403X

Éditeur :

Armand Colin, Société des études robespierristes

\section{Édition imprimée}

Date de publication : 1 juin 2005

Pagination : 47-55

ISSN : 0003-4436

\section{Référence électronique}

Anne Dion-Tenenbaum, « Martin Guillaume Biennais : une carrière exceptionnelle », Annales historiques de la Révolution française [En ligne], 340 | avril-juin 2005, mis en ligne le 15 juin 2008, consulté le 23 avril 2022. URL : http://journals.openedition.org/ahrf/2058 ; DOI : https://doi.org/10.4000/ahrf.2058

Ce document a été généré automatiquement le 23 avril 2022.

Tous droits réservés 


\title{
Martin Guillaume Biennais : une carrière exceptionnelle
}

\author{
Anne Dion-Tenenbaum
}

L'approche monographique est une des plus traditionnelles, mais aussi peut-être une des plus efficaces en histoire de l'art quand le domaine considéré est encore peu ou mal connu. Elle permet en effet, petit à petit, par le jeu des comparaisons, la mise en place de synthèses d'ensemble sûres, appuyées sur des faits précis. Les arts décoratifs de la Révolution et de l'Empire ne peuvent que bénéficier de tels travaux, encore trop embryonnaires. On connaît assez bien les grands ensembles hérités surtout du remeublement des résidences consulaires puis impériales (Malmaison, Fontainebleau [1], Compiègne, le Grand Trianon) ou du nouvel essor provoqué soit par la montée en puissance d'une bourgeoisie riche et cultivée, aimant les arts et le luxe sous le Directoire ${ }^{2}$, dont l'image emblématique est celle de Juliette Récamier (une partie du mobilier de son hôtel de la rue du Mont-Blanc, mobilier qui fit époque, est conservée au Louvre) ${ }^{3}$, soit par la naissance, un peu plus tard, d'une nouvelle noblesse (en témoignent encore aujourd'hui à Paris l'hôtel Beauharnais, actuelle ambassade d'Allemagne, et l'hôtel de Charost, résidence de Pauline Bonaparte, princesse Borghèse, actuelle ambassade de Grande-Bretagne). Mais les acteurs mêmes de ce renouveau, au premier rang desquels les ébénistes et les orfèvres, commencent tout juste à être étudiés dans le détail. Parmi eux se détache une figure singulière, singulière par son succès, par son esprit d'entreprise et son originalité dans le milieu parisien du commerce de luxe, celle de Martin Guillaume Biennais (1764-1843) ${ }^{4}$. Retracer sa carrière, c'est examiner le parcours d'un homme entreprenant, qui sut exploiter avec habileté les opportunités offertes par la suppression des corporations, puis par la renaissance du luxe sous le Premier Empire, et passer de la tabletterie à la petite ébénisterie et à l'orfèvrerie. Modeste tabletier à la veille de la Révolution, Biennais appartient à un milieu professionnel peu étudié ${ }^{5}$, que deux inventaires après décès successifs nous permettent ici de mieux cerner : celui de son prédécesseur Anciaux, en mai $1787^{6}$, puis celui de Biennais lui-même, à l'occasion de la mort de son épouse, en juillet $1789^{7}$. Pour les années révolutionnaires, les sources sont malheureusement rares ${ }^{8}$. Il faut attendre le Consulat, et l'organisation de la Maison du Premier Consul et 
surtout de l'Empereur ${ }^{9}$, dont Biennais devient le fournisseur attitré, pour avoir accès à des sources régulières, qui révèlent l'abondance et la variété de la production des ateliers de Biennais. À ces sources manuscrites, bien évidemment fondamentales, s'ajoute l'étude des œuvres conservées: ainsi peut-on tenter d'établir ce que fut la carrière de Biennais.

2 Première étape de cette carrière, Biennais est reçu maître tabletier à Paris, en septembre $1788{ }^{10}$. Bien qu'il ait réussi à pénétrer le milieu fermé des corporations, il est pourtant issu d'une petite paysannerie normande, sa famille se partageant entre les paroisses voisines d'Almenêche et de La Cochère, dans l'Orne. Son père laboureur meurt ${ }^{11}$, alors qu'il n'a pas encore huit ans. En 1782, le jeune Martin Guillaume réside à Argentan et il est domestique de l'abbesse de la prospère abbaye royale d'Almenêche, qui a une succursale dans cette ville ${ }^{12}$. En octobre 1785, il est dit tourneur à Argentan, et vend une maison héritée de sa mère, à La Cochère ${ }^{13}$. Il est probable que c'est à la protection de l'abbesse que le jeune homme doit cette formation artisanale, alors que tous ses frères et oncles sont laboureurs. Biennais se défait sans doute de son petit patrimoine avec le projet de quitter son sol natal ; c'est en tout cas sa dernière trace en Normandie.

3 On le retrouve à Paris, achetant le 20 avril 1788, pour 3000 livres ${ }^{14}$, un fonds de tabletterie rue Saint-Honoré, grâce notamment au petit capital produit par la vente de la maison maternelle. De tourneur, il est donc devenu tabletier, le tabletier étant, selon la définition donnée par les Tablettes de renommée, celui qui a «l'art de faire toutes sortes de pièces délicates au tour et autres menus ouvrages en ivoire, écaille et bois précieux tels que jeux de trictrac, dames, échecs, tabatières, peignes... ». Les statuts de la corporation des tabletiers ont été renouvelés par l'ordonnance de septembre 1741 : la communauté comprend dès lors les «maîtres et marchands peigniers, tablettiers, tourneurs, mouleurs, piqueurs, faiseurs et compositeurs de bois d'éventails, marquetteurs, tourneurs et tailleurs d'images d'yvoire et enjoliveurs de leurs ouvrages ». Ils ont le droit de fabriquer des bois d'éventail, des jeux, des dés, des croix, des tabatières, des peignes d'ivoire, d'écaille ou de buis. L'édit royal de 1776 les réunit aux luthiers et aux éventaillistes.

4 Le commerce acquis par Biennais est certes modeste, puisqu'il rachète à la veuve le fonds du tabletier Anciaux, installé en avril $1786{ }^{15}$, et mort dès mai 1787 sans avoir eu le temps de développer son affaire. La boutique est en revanche fort bien située; à l'écart du traditionnel quartier des tabletiers, à l'est des Halles (rues des Arcis et de la Tabletterie), Biennais est proche des Tuileries, dont il deviendra quelques années plus tard le fournisseur, et au centre du quartier des marchands-merciers. Est-ce ce voisinage qui l'incitera à reprendre à son compte certaines spécialités de cette profession, lorsqu'elle déclinera après la Révolution? Jean-Baptiste Latte, tabletier rue des Arcis, qui pourrait avoir été le maître de Biennais à son arrivée à Paris, a probablement orienté cet achat; c'est lui en effet qui est chargé de l'expertise des marchandises, lors du décès d'Anciaux en 1787, puis lors du rachat par Biennais en 1788 ${ }^{16}$.

5 Un an après son mariage, Biennais se retrouve veuf et père d'une petite fille. La mort prématurée de son épouse nous vaut un précieux inventaire après décès ${ }^{17}$. L'estimation - à nouveau confiée à Latte - des marchandises, ustensiles et outils, se monte à 5400 livres (au lieu de 1000 à la mort d'Anciaux). L'inventaire nous renseigne par ailleurs sur la variété de la production, qui correspond fidèlement aux différentes facettes du 
métier codifiées par les statuts corporatifs. Sont ainsi énumérés de menus objets usuels (peignes, brosses à dents, boutons, porte-crayons...), quelques crucifix ou chapelets, des boîtes, tabatières et bonbonnières en corne ou en écaille. L'essentiel est néanmoins constitué par les jeux: damiers, échiquiers, dominos, jeux de solitaires, de trictracs, bilboquets, totons... Biennais pratique la sous-traitance puisqu'il doit 101 livres 16 sols à l'éventailliste Enguerhard, 58 livres au tabletier Delacroix, et la somme plus importante de 571 livres au tabletier Pierre Duménil, coauteur de l'inventaire. Bien qu'il s'intitule parfois tabletier et éventailliste, les éventails ne sont pas sa spécialité ; il abandonnera plus tard quasiment cette production, au contraire des jeux ou des bonbonnières fabriqués tout au long de sa carrière. Quelques meubles d'enfants, isolés dans son stock, pourraient revenir à Delacroix ou à Duménil. Deux tours constituent le gros outillage, pour lui et l'apprenti qu'il a engagé.

On ne sait comment Biennais traverse les années révolutionnaires. En 1790, puis en 1791, il passe une annonce dans Annonces, affiches et avis divers ${ }^{18}$. En décembre 1790, il signe un nouveau bail pour la location de deux boutiques, celle qu'il occupait jusque-là à droite de la porte cochère du 510 rue Saint-Honoré, et qu'il sous-loue aussitôt à un marchand-brossier, et celle de gauche, plus spacieuse, dans laquelle il s'installe. Ses affaires ne semblent pas trop pâtir des événements, puisqu'il est en mesure d'acheter, en juillet 1795, une belle maison à Choisy-le-Roi ${ }^{19}$, profitant peut-être des gains tirés de la clientèle des nouveaux riches, dont le luxe s'affiche sous la Convention thermidorienne. Il semble donc que ces années où le commerce de luxe a traversé une passe très difficile jusqu'au Directoire n'ont pas été, pour lui, financièrement douloureuses. Son commerce s'est-il malgré tout maintenu justement parce qu'il l'avait limité à des objets de consommation plus courante? On ne sait, mais il est désormais bien installé, et son commerce bien établi.

7 Les plus anciennes œuvres conservées sont de petites coiffeuses de lit, des jardinières, des bidets de voyage, ou parfois des nécessaires de voyage ${ }^{20}$. Ces pièces sont en général signées "Biennais Md Tabletier ébéniste au Singe violet rue Honoré n 511 », comme cette jardinière à douze pans, en acajou, reposant des quatre pieds à griffes, certainement bien représentative de la production de ces débuts (musée du Louvre, fig. 17). Lorsqu'il s'agit de nécessaires, ils sont garnis de pièces d'orfèvrerie fournies par l'orfèvre Marie Joseph Gabriel Genu, ou encore par les spécialistes du couvert Lorillon ou Naudin, aux poinçons de titre et de garantie postérieurs à 1798 .

8 Le singe ${ }^{21}$, mentionné dans la signature, se retrouve dans l'en-tête des factures. On n'en conserve pas à l'adresse 511 rue Honoré. Les premiers portent le numéro 119 rue Honoré ou Saint-Honoré ${ }^{22}$. Son en-tête montre donc un singe accroupi devant une table de jeu, et secourant les dés dans un cornet, outre une composition réunissant un damier, un éventail, un jeu de trictrac, un crucifix et des pions, donc un échantillonnage représentatif de sa production; il est dit marchand tabletier et éventailliste, la suppression des corporations ayant banni le terme de maître. Une énumération d'articles explicite ce que suggère cette pittoresque imagerie. Les en-têtes successifs reflètent l'élargissement rapide des activités de Biennais; il s'ajoute bientôt (pour la même adressse) un paragraphe sur les meubles, qui accompagne l'allongement des titres de Biennais, désormais non seulement tabletier et éventailliste, mais également ébéniste. Enfin est introduite l'orfèvrerie, et la présentation de la production devient tripartite : tabletterie, ébénisterie, orfèvrerie. L'orfèvrerie va progressivement évincer la tabletterie, et, parallèlement, le singe est de plus en plus marginalisé, pour 
finalement disparaitre au profit d'une composition architecturale antiquisante, dans le style de Percier (fig. 18) ; les symboles impériaux affirment clairement que Biennais est l'orfèvre de l'Empereur, et les titres sont de plus en plus ronflants, jusqu'à devenir, «Orfèvre de Leurs Majestés impériales et royales, de Leurs Majestés le roi de Hollande et de Westphalie ».

Ce passage à l'orfèvrerie, qui marque une évolution capitale dans la carrière de Biennais, a été semble-t-il en grande partie la conséquence indirecte des changements politiques. En effet, dès le coup d'État des 18 et 19 brumaire, Biennais a su s'attirer la faveur de Napoléon Bonaparte; c'est ce que rapporte Taillandier, dans le discours prononcé lors des funérailles de Biennais, en 1843 : «Le général Bonaparte, à son retour d'Égypte, voulut monter sa maison. Il ne possédait encore d'autre fortune que sa gloire ; aussi, les négociants auxquels il s'adressa d'abord refusèrent de lui vendre à crédit. Biennais eut plus de confiance dans l'étoile du jeune général, et il lui fit des fournitures considérables sans s'occuper de l'époque où elles lui seraient remboursées. Napoléon fut reconnaissant de cette marque de confiance, et, devenu empereur, il prit Biennais pour son orfèvre $"{ }^{23}$. On peut supposer que les articles de voyage proposés par Biennais (nécessaires, bidets...) étaient propres à séduire un général souvent en campagne ; quant à la coquette et mondaine Joséphine, elle pouvait trouver chez le tabletier des articles de toilette ou encore des jeux. Quoi qu'il en soit, son commerce prend désormais un nouvel essor, essor dû certes à ce patronage prestigieux, mais aussi à l'extension de l'activité de Biennais, qui bouscule les catégories d'activité héritées de l'Ancien Régime encore très présentes dans l'organisation et la séparation des métiers parisiens touchant aux arts décoratifs.

En 1801 ou 1802, Biennais a fait insculper un poinçon, pour lequel il choisit à nouveau un singe comme symbole. Les plaques d'insculpation ne permettent pas de préciser la date exacte. Lorsqu'en novembre 1801, il livre à Joséphine un service à thé, ce dernier est exécuté par Genu, et est signé "Biennais au Singe violet », sans que soit encore introduit de titre d'orfèvre ${ }^{24}$. Il s'agit de pourvoir la table du Premier Consul d'un service de table de deux cents couverts. On peut donc supposer que si cette commande lui est adressée, c'est qu'il est déjà tout au moins marchand d'orfèvrerie. Peu après, Biennais livre au Premier Consul une athénienne (ou lavabo) pour les Tuileries (musée du Louvre, fig. 19), signée "Biennais Orfèvre du Premier Consul », même si le bassin et l'aiguière portent encore le poinçon de Genu. Le modèle de cet élégant trépied d'inspiration antique peut être attribué à Percier ; le vocabulaire décoratif, en partie lié au thème de l'eau (dauphins, cygnes), est néoclassique (palmettes, fleurons, feuilles d'acanthe, frise de postes...). À l'exposition des produits de l'industrie de 1806, si Biennais présente, outre des pièces d'orfèvrerie, un nécessaire et une pièce d'ébénisterie, c'est néanmoins dans la seule section orfèvrerie, que le jury commente ses œuvres et lui accorde une médaille d'or ${ }^{25}$. Percier et Fontaine publient, dans leur fameux Recueil de décorations intérieures (fig. 20), un pot à oille commandé par Joséphine à Biennais, présenté à cette exposition de 1806 : sur le bas-relief de la panse, deux femmes, un genou à terre, encadrent des cornes d'abondance et le chiffre J couronné ; deux bustes de femme soutiennent chaque anse; enfin sur le couvercle, une femme assise (Cybèle ou Cérès), tenant un vase chargé de fruits, est entourée de deux enfants.

11 Biennais a donc rapidement jugé trop étroit le cadre des activités traditionnelles d'un tabletier, comme l'expose le discours prononcé lors de ses funérailles : «il ne tarda pas à voir que cette industrie était trop restreinte et que sa destinée l'appelait à exercer un 
art qui devait offrir à sa haute intelligence des ressources bien autrement étendues. Il embrassa la profession d'orfèvre... ». Ce glissement à l'orfèvrerie était assez logique ${ }^{26}$. Pour les nécessaires, il est désormais possible à Biennais, jusque-là confiné dans l'exécution du contenant, à savoir le coffret, d'ailleurs très élaboré avec ses cases aux profils exacts des objets destinés à y être rangés, et d'une partie du contenu (étuis en buis, manches de rasoirs ou couteaux en nacre, brosses à dents, mètres, dés en ivoire...) (fig. 21), de s'occuper de toute la garniture. Biennais peut donc maîtriser toutes les phases de sa production, ce qui ne l'empêchera pas, jusqu'à la mort de l'orfèvre Genu en 1810 , d'avoir recours à ce désormais confrère. Biennais a bien compris que le travail de l'argent et du vermeil était plus propre à accompagner la renaissance du luxe et la volonté d'une vie de Cour prônée par Napoléon. En outre, il devait être assez flatteur d'accéder aux prérogatives d'un des plus prestigieux corps marchands d'Ancien Régime, alors qu'il était interdit aux tabletiers avant la Révolution d'utiliser l'or ou l'argent, sinon à la réquisition des orfèvres.

Biennais sait s'adapter avec souplesse à la volonté impériale. Lorsque Napoléon institue la Légion d'honneur, puis l'ordre de la Couronne d'Italie, Biennais se met à fabriquer des décorations, et il est tout prêt à assurer cette fabrication, lorsque les frères de l'Empereur l'imitent, avec l'ordre de la Couronne de Westphalie, l'ordre de l'Union ou des Deux-Siciles. Il écrit qu'il a «des ateliers outillés et montés pour aller vite à cette besogne $"{ }^{27}$. Si d'autres orfèvres assurent également cette fabrication, Biennais semble avoir l'exclusivité des colliers ${ }^{28}$. Très vite, Biennais a fait entrer dans sa production les armes blanches; épées ou sabres d'apparat sont livrés à Napoléon ou à ses frères, notamment au fastueux Jérôme de Westphalie. Pour le sacre, l'orfèvre de l'Empereur a reçu la prestigieuse commande des insignes impériaux (couronne, globe, main de justice, sceptre), et de la restauration des ornements conservés d'Ancien Régime.

13 Sur l'organisation des ateliers, les sources manquent. Biennais a acheté l'immeuble du 121 rue Saint-Honoré, dès le 31 décembre 1803, pour la somme de 124100 francs ${ }^{29}$. D'après une enquête menée par la préfecture de police en 1807, période de crise, soixante ouvriers sont employés par l'entreprise ; à la même date, l'orfèvre Odiot en emploie cinquante et l'ébéniste Jacob-Desmalter cinquante à soixante. Selon une notice publiée en 1821, peu avant le retrait des affaires de Biennais ; " ...chaque pièce, depuis l'instant où elle commence à être mise en œuvre, jusqu'à celui où elle sera terminée, ne sort pas de ses ateliers : en effet, toutes les mains nécessaires à la fabrication et à l'entière perfection des ouvrages qu'on y fabrique s'y trouvent réunies. Pendant nombre d'années, cette maison a occupé, dans son intérieur, de 150 à 180 ouvriers, sans compter ceux qu'elle employait au dehors " ${ }^{30}$. Lorsqu'il se retire, Biennais loue à son successeur Cahier la boutique du rez-de-chaussée, la cave, les écuries, et tout le premier étage (sur rue et sur cour) ${ }^{31}$, qui sont donc les lieux utiles à son activité. La faillite de ce dernier, en 1828 , entraîne une mise sous scellés ${ }^{32}$, qui nous vaut un inventaire, dont on peut sans doute tirer des enseignements sur la topographie des lieux du temps de Biennais. L'inventaire énumère dix ateliers : un atelier du balancier, une fonderie, un très important atelier des orfèvres, un atelier des bijoutiers et un petit attenant, un de tourneur, un des polisseuses et brunisseuses, celui de ciselure, celui du gainier et enfin celui des ébénistes.

14 C'est donc une énorme entreprise que dirige Biennais, et le nombre des pièces conservées atteste l'importance de sa production. Les Bonaparte et les Beauharnais sont tous clients chez Biennais. Joséphine, Hortense, Eugène, vice-roi d'Italie, Élisa, 
grande duchesse de Toscane, Louis, roi de Hollande, Jérôme de Westphalie, le prince Camille Borghese ont sur leur table des services d'argent et de vermeil, qui sortent de ses ateliers. Le sucrier de la reine Hortense (donné au musée du Louvre sous réserve d'usufruit ; fig. 22) témoigne du luxe de ces services. En forme de coupe couverte, porté par une colonne balustre, il repose sur un plateau orné de trois sphinges, entourées des cuillers dressées tout autour en couronne. Grâce à l'impulsion donnée par ces exemples princiers, Biennais bénéficie d'une large clientèle de généraux (Duroc...), maréchaux (Bessière, Berthier...), officiers de la Couronne (le chambellan Rémusat). À la fin de sa carrière, dans les premières années de la Restauration, Biennais travaille beaucoup pour une clientèle étrangère, et livre un énorme service pour Nicolas Pavlovitch, puis un autre pour le grand duc Michel, de même qu'une toilette pour l'épouse du roi de Wurtemberg. La production la plus courante nous échappe, mais elle reprend, en les simplifiant, en argent (et non en argent doré) les formes et l'ornementation de ces commandes prestigieuses.

La Restauration n'a pas marqué la fin de l'activité de Biennais, même s'il avait pu bénéficier, à certains moments de sa carrière, d'opportunités dues aux changements politiques, et s'il apparaissait comme très lié au régime impérial, allant même jusqu'à acheter une propriété aux Lavalette en 1816. Mais s'il cessa son activité, ce fut en définitive pour prendre sa retraite. Biennais se retire fin 1821. Il a pour descendance six filles, que, hormis l'aînée (née du premier mariage) qui a épousé un joaillier, il n'a pas marié dans le milieu de l'orfèvrerie ou de la tabletterie. Il vend donc son affaire à un concurrent, bien en cour auprès des Bourbons, Jean-Charles Cahier; ce dernier gèrera assez mal ses affaires et fera définitivement faillite en 1830. On peut donc dire que la maison créée par Biennais a disparu avec lui, au contraire d'autres maisons dirigées par de solide dynasties, nées avant ou pendant la Révolution (on pense en particulier, dans le milieu de l'orfèvrerie, à celle des Odiot). Se pose ainsi en définitive la question des causes du succès de Biennais. Elles semblent essentiellement dues à son talent de fabricant et "d'inventeur » (les fameux nécessaires notamment, qui firent tant pour sa renommée et son succès commercial), talent conforté par son aptitude de gestionnaire. Saisissant toutes les opportunités, sachant répondre à la demande de nouveaux milieux et saisir en même temps les évolutions du goût (son médaillier pour Vivant-Denon), il semble avoir aussi parfaitement maîtrisé les difficultés d'une époque où le commerce subit sans cesse les contrecoups des conflits intérieurs et extérieurs. Il apparait ainsi, sous quelque angle qu'on aborde sa carrière, comme une personnalité singulière, celle d'un self made man à qui sa maison, privée de son génie, ne survécut pas.

\section{NOTES}

1.Sur Fontainebleau, voir notamment J.-P. SAMOYAULT, cat. de l'exp. Le Mobilier du général Moreau, un ameublement à la mode en 1802, Fontainebleau, 1992, et Fontainebleau, musée national du château. Meubles entrés sous le Premier Empire, 2004.

2.Cf. cat. de l'exp. Au temps des merveilleuses. La société parisienne sous le Directoire et le Consulat, Paris, musée Carnavalet, 2004. 
3.G. LEDOUX-LEBARD, « La Chambre de Madame Récamier au Louvre, un apogée du style consulaire ", L'Estampille/L'Objet d'art, mars 1994, pp. 64-89; et Nouvelles acquisitions du département des Objets d'art, 1990-1994, 1995, pp. 216-226.

4.Voir A. Dion-TenEnBAum, cat. de l'exp. L'Orfèvre de Napoléon, Martin-Guillaume Biennais, Paris, musée du Louvre, 2003-2004.

5.Voir néanmoins D. AlcoufFE, « Les Tabletiers parisiens au XVIIIe siècle », Métiers d'art, $\mathrm{n}^{\circ}$ 54-55, «La tabletterie », décembre 1994- mars 1995, p. 22-26.

6.A. N., MC XXXV/895, 11 mai 1787, inventaire après décès ; voir aussi Y 15298, mise sous scellés après le décès de Claude-Louis Anciaux, le 6 mai 1787.

7.Ibid., LXII/654, 31 juillet 1789, inventaire après décès de Rosalie Maheu.

8.Quelques factures sont conservées dans la série $\mathrm{T}$ des saisies révolutionnaires, mais les y retrouver est fort aléatoire.

9.La série 02, à partir de l'an XIII, révèle l'abondance et la diversité des fournitures de Biennais à la Maison de l'Empereur : nécessaires, jeux, petits meubles, services de table, objets de bureau, épées, décorations...

10.A-N-, Y 9334, registre de réception.

11.Guillaume Biennais meurt le 29 février 1772 (A-D-Orne, état-civil de La Cochère, et notaire d'Almenêche, 10 mai 1772).

12.Ibid., 3E2 006/15, registre paroissial de Saint-Martin d'Argentan. Biennais représente un parrain absent lors d'un baptême, le 18 décembre 1782, et il est alors dit domestique de Jeanne-Marie de Eame, abbesse de l'abbaye royale d'Almenêches.

13.Ibid., 4E74/450, maître Féval, 20 octobre 1785, vente de M.-G. Biennais à Louis-Pierre Lemoul.

14.Acte sous seing privé analysé dans l'inventaire après décès de Rosalie Maheu. 15.Claude-Louis Anciaux avait été reçu maître tabletier luthier éventailliste en mai 1778.

16.Il est également témoin lors du mariage de Biennais avec Rosalie Maheu (A-N-, MC, CX/522, 2 juin 1788, contrat de mariage).

17. Cité note 4 .

18.1790, p. $1006 ; 1791$, p. 2162.

19.A-D-Val-de-Marne, CXXVII/58, 10 thermidor an III, achat à N.-Fr. Bellanger. La maison est achetée pour le prix de 80000 livres, dont 25000 sont aussitôt acquittés en assignats sur les domaines nationaux ; solde et quittance le 24 thermidor an IV. 20.On peut citer notamment une jardinière (musée du Louvre, Objets d'art), une coiffeuse de lit avec son nécessaire au chiffre JB de Joséphine (musée national de Malmaison), ou un nécessaire provenant du maréchal Bessières (Paris, musée des Arts décoratifs)...

21.Il faut signaler qu'un autre tabletier, François Vaugeois, attesté à partir de 1778, auquel succède son fils Bernard-François en décembre 1793, avait une enseigne curieusement similaire : «Au singe vert». Vaugeois fournit sous l'Empire essentiellement des jeux et tables à jeu.

22.La numérotation change en effet : le 509 ou 510 devient le 119, et le 511 (où Biennais va finalement se fixer) successivement le 121, 283 puis 173.

23. Moniteur universel, 30 mars 1843, p. 613. Le député Taillandier, fils d'avoué et avocat lui-même, était lié depuis plus de vingt ans à deux des gendres de Biennais, Foussier et Berger, également avoués ; on peut donc supposer qu'il était bien informé sur l'histoire de Biennais. 
24.Le service (coll. part.) porte également la signature « Orfèvre de Sa Majesté l'Empereur et Roi à Paris », mais elle est évidemment postérieure à la livraison, et a probablement été apposée en même temps que le chiffre J couronné de Joséphine. 25. Notice sur les objets envoyés à l'exposition des produits de l'industrie française, rédigées et imprimées par ordre de S.E.M. de Champagny, ministre de l'Intérieur, an 1806, 1806, n 563. 26.Logique, mais non obligatoire. Le principal rival de Biennais pour les nécessaires, Pierre-Dominique Maire (vers 1763-1827), installé rue Saint-Honoré face à l'Oratoire, s'intitule « marchand d'orfèvrerie et fabricant de nécessaires » et il fera toujours exécuter les pièces d'orfèvrerie à l'extérieur, notamment par Beaufort ou par Blaquière, " orfèvre garnisseur ", fournissant les pièces de nécessaires.

27.Paris, musée de la Légion d'honneur, lettre de Biennais, s.d. [août 1811].

28.Le musée de l'Armée conserve un collier et le musée de la Légion d'honneur deux. 29.L'immeuble, vendu aux enchères, est acquis par jugement de l'audience de criée du tribunal de première instance de la Seine, le 9 nivôse an XII ; il n'y a donc pas d'acte notarié.

30.Bazar parisien, 1821, pp. 37-38. Plus tard, dans son rapport sur l'Exposition universelle de 1851, Honoré d'Albert, duc de Luynes (Industrie des métaux précieux..., Paris, 1854, p. 58) évoque le nombre peu vraisemblable -mais souvent repris par la bibliographie postérieure- de 500 à 600 ouvriers.

31.Arch. de Paris, DQ18208, p. 110, enregistrement du contrat de location sous seing privé.

32.Ibid., D1U1 art. 121, mise sous scellés, 29 novembre 1828 (document signalé par Mme Brigitte Lainé).

\section{RÉSUMÉS}

Cet article retrace les principales étapes de la carrière de Martin Guillaume Biennais. Issu de la petite paysannerie normande, Biennais, reçu maître tabletier à Paris en 1788, s'installe rue SaintHonoré. L'inventaire, dressé lors de la mort de son épouse en 1789 , donne une première idée de son commerce. Homme entreprenant, il sait très habilement profiter de la suppression des corporations pour étendre ses activités à l'ébénisterie, puis sous le Consulat, à l'orfèvrerie. La faveur du Premier Consul puis Empereur, dont il devient l'orfèvre attitré, favorise son essor. Mais cette irrésistible ascension est sans suite : Biennais n'a pas de successeur, et vend fin 1821 son affaire à Cahier, qui fera faillite en 1830.

Martin Guillaume Biennais, a carrer of exception. This article is about the career of MartinGuillaume Biennais. Biennais, one of the small peasantry class, went to Paris prior the Revolution and established himself as a maitre tabletier, whose activity is primarily known from the death inventory of his first wife. He was soon extending his business after the suppression of corporations, first as an ébéniste, and, during the Consulat, as a goldsmith. Much favoured by Napoléon, who gave him an imperial appointment, he came to a climax during the Empire. But his firm, which was sold to Cahier in 1821, was not to be continuing after him, for in 1830 his successor went bankrupt. 
INDEX

Mots-clés : Tabletier, orfèvre, ébéniste, corporation, singe, poinçon, nécessaire, Biennais (Martin-Guillaume), Genu (Marie-Joseph-Gabriel), Cahier (Jean-Charles), ateliers

\section{AUTEUR}

\section{ANNE DION-TENENBAUM}

Conservateur en chef au département des Objets d'art du musée du Louvre, 101, rue de Rivoli 75001 Paris 